\title{
Analysis of Factors Influencing the Prognostic Significance of Hyponatremia in Peritoneal Dialysis Patients
}

\author{
Luis Bravo González-Blas ${ }^{\mathrm{a}}$, Leticia García-Gago’, Daniela Astudillo-Jarrín ${ }^{\circ}$ \\ Catuxa Rodríguez-Magariños , Antía López-Iglesias a , Teresa García Falcón ${ }^{\text {a }}$,Ana \\ Rodríguez-Carmona ${ }^{\mathrm{a}}$,Miguel Pérez Fontán ${ }^{\mathrm{a}, \mathrm{b}}$
}

${ }^{a}$ Division of Nephrology, University Hospital A Coruña, A Coruña, Spain

${ }^{\mathrm{b}}$ Professor of Medicine, Faculty of Health Sciences, University of A Coruña, A Coruña, Spain

\begin{abstract}
Background:

The evidence linking low serum sodium levels with the risk of mortality in peritoneal dialysis (PD) patients is controversial. Considering the different mechanisms contributing to hyponatremia in these patients, it is conceivable that the prognostic significance of this factor may vary, according to the clinical setting.
\end{abstract}

Methods:

Following a retrospective, observational design, we analyzed the association between hyponatremia and mortality in 748 patients incident on PD. We applied multivariate strategies of analysis, with the main objective of identifying subgroups of patients in whom hyponatremia could sustain different degrees of association with mortality (main outcome variable). For this purpose, we performed preliminary analyses to: (1) disclose predictors of serum sodium levels before and after (mean of first 3 months) initiation of PD (main study variable) and (2) investigate the overall prognostic significance of hyponatremia, in our patients.

\begin{abstract}
Results:
Comorbidity, hypoalbuminemia, and lower glomerular filtration rate (GFR) were main predictors of hyponatremia. Use of icodextrin was another inverse correlate of serum sodium, and the only consistent predictor of a decline of natremia, once PD was started. Multivariate analysis confirmed early hyponatremia as an independent marker of survival. However, stratified analyses showed that this association was most apparent in specific subsets, namely, hypoalbuminemic, more anemic patients with higher baseline levels of GFR and C-reactive protein and faster peritoneal solute transport rates. Other factors potentially reinforcing the prognostic significance of hyponatremia included lower lean body mass levels, nonprescription of reninangiotensin-aldosterone system antagonists, and use of icodextrin-based PD solution. On the contrary, baseline overhydration or categorization by classic predictors of mortality (age, comorbidity, diabetes) did not appear to influence the risk pattern associated with lower serum sodium levels.
\end{abstract}

\section{Conclusions:}

Our results suggest that hyponatremia performs as a consistent correlate of the risk of mortality mainly in PD patients manifesting direct or indirect signs of inflammation and wasting, while this association is not apparently linked to the presence of overhydration or nominal, preexisting comorbid conditions 


\section{Keywords}

Peritoneal dialysis; Sodium; Hyponatremia; Mortality; Inflammation; Malnutrition; Overhydration

\section{Introduction}

Patients with chronic kidney disease (CKD) present markedly increased rates of mortality, related both to associated clinical conditions (usually assessed from age, presence of diabetes, and comorbidity scores) and to the adverse consequences intrinsic to CKD and its management [1]. The complex pathogenesis of CKD-related mortality complicates the generation of models predicting this outcome, fed by the countless array of potential markers reported in the literature [2]. Fluid and electrolyte disorders are extremely frequent in CKD, and their influence on the outcome of these patients has been extensively investigated [3, 4]. In particular, the correlation between disorders of sodium concentration (mainly hyponatremia, but also hypernatremia) and mortality at different stages of CKD has been a subject of great interest [5]. A link between these 2 complications is generally accepted, although it is often unclear in which proportion hyponatremia is a risk marker and a cause of mortality, in this setting.

In the particular case of patients undergoing chronic peritoneal dialysis (PD), the evidence linking hyponatremia and mortality is limited and controversial, with some studies reporting a significant association [6-8], which has not been observed by others [9, 10]. Admitting that there are different potential mechanisms leading to hyponatremia in these individuals [11, 12], it is conceivable that at least part of these discrepancies may be related to different characteristics of PD patients and practices. This hypothesis suggests the convenience of identifying clinical subsets in which serum sodium could perform more or less efficiently as a risk marker of PD-related mortality.

We have undertaken a retrospective, observational study of a relatively large population of patients incident in PD therapy, with the primary objective of identifying specific clinical subsets modulating the association between early hyponatremia and mortality during follow-up. Our main interest was to clarify the relative role of 3 main mechanisms of hyponatremia in PD patients, namely overhydration, wasting/inflammatory states, and the conditions of drug and dialysis prescription. To accomplish these goals, we performed preliminary analyses to (1) disclose clinical predictors of serum sodium levels before and after initiation of PD and (2) appraise the overall correlation between serum sodium .levels and survival, in our setting (secondary objectives).

\section{Methods}

\section{General Design}

Following a retrospective, observational design, we analyzed the prognostic significances of serum sodium levels preceding initiation of PD (preNa), mean of values recorded during the first 3 months of PD therapy (mean serum sodium [mNa]), and changes in sodium levels between the aforementioned periods. Our main objective was to disclose particular subsets in which mNa could 
bear a differentiated prognostic value. The main outcome variable was mortality during follow-up on PD. The study setting was a tertiary university hospital, sustaining a mean prevalent population on PD of 120 patients, and a mean incidence of 35 patients per year. We applied multivariate strategies of analysis to control for other variables with an influence on the main outcome. Secondarily, we explored the clinical spectrum of predictors of preNa, $\mathrm{mNa}$, and changes in sodium levels.

The study complied with the principles of the Declaration of Helsinki and the ethical requirements of our center for observational, retrospective studies. Oral informed consent was obtained from all patients available at the time of initiation of the study.

\section{Population}

For this study, we selected patients initiating PD therapy between January 1991 and June 2018, fulfilling the following inclusion/exclusion criteria:

-- Adult patients (> 18 years of age)

- Incident on PD

-- Patients alive after 90 days on PD therapy

-- At least 1 estimation of serum sodium levels in the 2 months preceding PD start available

- At least 1 estimation of serum sodium levels during the first 3 months after inception of PD available

-- Information on preexisting comorbidity, residual kidney function (RKF) an drug, and dialysis prescription at the start of PD available

-- Oral informed consent provided (only patients active at the initiation of the study).

\section{Study Variables}

$\mathrm{mNa}$ level was the main study variable and was estimated as the mean of available serum $\mathrm{Na}$ levels recorded during the first 3 months after initiation of PD (median 3 estimations, range 1-5). preNa (last $\mathrm{Na}$ value before initiation of PD) and changes in sodium levels after inception of PD (difference $\mathrm{mNa}-$ preNa) were secondary study variables. Serum sodium levels were estimated using the same ionselective electrode method during all the study period. Mortality was the main outcome variable. By definition, all patients had a minimal survival of 3 months on PD. Patients were censored in case of kidney transplant, change to hemodialysis therapy, or loss to follow-up. 
Control variables included: dialysis vintage (PD started before/after the end of 2004), age, gender, comorbidity (Charlson's comorbidity score), history of heart disease, diabetes, malnutrition (standard subjective global assessment), body mass index (BMI; weight/height2), glomerular filtration rate (GFR; mean of the renal clearances of urea and creatinine), plasma albumin, serum C-reactive protein (CRP; immunoturbidimetry), blood hemoglobin, D/P creatinine ratio at $240 \mathrm{~min}$ during the baseline peritoneal equilibration test (PET; D/Pcrea), standardized ultrafiltration during the baseline PET (available in only 241 patients with a baseline PET performed with 3,86\% glucose-based dialysate), modality of PD, use of icodextrin for the long dwell, and treatment with loop diuretics (we applied an arbitrary equivalence of $10 \mathrm{mg}$ of torasemide $=40 \mathrm{mg}$ of furosemide), renin-angiotensin-aldosterone system (RAAS) antagonists, psychotropic drugs (antidepressives and/or antipsychotics), and immunosuppressives (including steroids, active during the preceding year and/or at the start of PD therapy). In a limited number of patients (those starting PD after early 2010; $n=180$ ), we scrutinized body composition at the time of the baseline PET, using a bioimpedance analysis device (BCM, Fresenius, Germany). We recorded lean body mass and fat mass (normalized for body surface), total overhydration, and ratio of extracellular to intracellular water. All the control variables were recorded at baseline, except PET and bioimpedance analysis (performed 4-6 weeks after initiation of PD), and treatment with icodextrin, loop diuretics, RAAS antagonists, and psychotropic drugs, for which we requested an active prescription during at least 2 of the first 3 months on PD.

\section{Strategy of Analysis}

Our strategy of analysis followed 3 steps:

1. We first investigated predictors of preNa, $\mathrm{mNa}$, and changes in sodium levels after PD was started. For this purpose, we applied univariate (Spearman's correlation coefficient, Student $t$ test and ANOVA) and multivariate (stepwise multiple regression) strategies. For multivariate analyses, we explored only first-order interaction terms. Diabetes and heart disease were explored either as independent variables or included in the global Charlson's score. Separate analyses were generated for variables available in a limited number of cases (body composition and ultrafiltration during the baseline PET).

2. Second, we performed an exploratory analysis of variables predicting mortality, searching for an adjusted effect of the main study variables. We first identified univariate predictors of mortality (Kaplan-Meier, log rank test), generating a reference multivariate model (Cox regression), oriented to estimate an adjusted association between preNa, $\mathrm{mNa}$, and changes in sodium levels, on one side, and mortality, on the other. Again, only first-order interaction terms were explored, and stratified analyses were generated for significant terms. Similarly to the previous step, we generated specific models for variables available in a limited number of cases.

3. Finally, we explored control variables with a clinical interest, but without a demonstrated independent association with patient survival, searching for specific subsets in which $\mathrm{mNa}$ levels could present a differentiated association with mortality. For this purpose, we used, again, stratified analyses, obtaining adjusted estimations (Cox) of the association between mNA and mortality in each explored subset.

We categorized numerical variables according to their median values, except in 2 specific cases (plasma albumin, GFR), in which we decided to fit categorization to clinical significance. We used SPSS 19.0 software for data management and analysis. 


\section{Results}

Overview

Eight hundred and seventy-seven patients started PD during the study period. The main causes of exclusion from the study were insufficient follow-up on PD $(n=61)$ and inadequate data on GFR $(n=29)$ and/or serum sodium levels before/after initiation of therapy $(n=23)$. Overall, 748 patients were included in the analysis. The main characteristics of the study population at initiation of PD are displayed in Table 1. The distribution of causes for CKD was standard. Table 2 shows plasma sodium levels before and after PD inception. Only 24 patients (3.2\%) presented mNa levels $>143 \mathrm{mM} / \mathrm{L}$, and only $4(0.5 \%)$ had a $\mathrm{mNa}>145 \mathrm{mM} / \mathrm{L}$; consequently, no further attention was dedicated to hypernatremia. Followup on PD was $31.6 \pm 25.7$ months. At the end of followup, only 77 patients were active on PD, while 346 patients (46.3\%) had died, 209 (27.9\%) had undergone a kidney transplant, $94(12.6 \%)$ had been switched to hemodialysis, $5(0.7 \%)$ suspended PD after partial recovery of kidney function, and 17 (2.3\%) were lost to follow-up. Death was mainly due to cardiovascular events $(n=159)$, infections $(n=84)$ and dialysis withdrawal $(n=42)$.

\section{Correlates of Plasma Sodium Levels}

Multivariate analysis disclosed comorbidity (Charlson's score), hypoalbuminemia, lower GFR, and a lower dose of loop diuretics as independent predictors of lower preNa levels (Table 3). Diabetes and a history of heart failure performed as convenient surrogates of Charlson's score, at the time of modeling preNa (Table 3 ).

The primary multivariate model for prediction of $\mathrm{mNa}$ identified Charlson's score, hypoalbuminemia, lower GFR, a more severe degree of anemia, and use of icodextrin for the long dwell as independent predictors of lower $\mathrm{mNa}$ levels (Table 4). Again, inclusion of diabetes and heart failure, rather than Charlson's score, improved marginally the quality of the model.

Older patients and those on loop diuretics tended to experience a decrease in serum sodium after initiation of PD but, in practical terms, icodextrin-based PD was the only consistent predictor of such an evolution (Table 5).

\section{Correlation between Serum Sodium Levels and Mortality: General Model}

Table 6 and Figure 1 display significant univariate predictors of mortality. The best multivariate model (Table 6) included well-known markers including age, Charlson's score, plasma albumin (or serum CRP), blood hemoglobin, GFR, and D/Pcrea at the start of PD. In addition, both preNa and $\mathrm{mNa}$ performed as independent predictors of mortality during follow-up, while the time course of serum sodium after initiation of PD did not show such an effect. Plasma albumin, serum CRP, blood hemoglobin, and GFR presented significant interaction with $\mathrm{mNa}$ levels, while D/Pcrea was close to significance (Table 6). The results of the subsequent stratified analyses will be presented in the next paragraph.

In separate models, ultrafiltration during the baseline PET did not perform as an independent predictor of mortality. Regarding body composition analysis, we observed only a minor, nonsignificant trend $(p=0.089)$ to an inverse association between lean body mass and the main study outcome. 


\begin{tabular}{|c|c|c|c|c|c|c|}
\hline & Overall & $\begin{array}{l}\text { Lowest quartile } \\
(<135.3 \mathrm{~mm} / \mathrm{L})\end{array}$ & $\begin{array}{l}\text { Second quartile } \\
(135.3-137.8 \mathrm{~mm} / \mathrm{L})\end{array}$ & $\begin{array}{l}\text { Third quartile } \\
(137.8-139.8 \mathrm{~mm} / \mathrm{L})\end{array}$ & $\begin{array}{l}\text { Highest quartile } \\
(>139.8 \mathrm{~mm} / \mathrm{L})\end{array}$ & $p$ value \\
\hline Age, years, mean (SD) & $59.4(15.4)$ & $60.8(15.3)$ & $59.5(16.1)$ & $60.1(15.0)$ & $57.8(15.2)$ & 0.25 \\
\hline Gender, males/females, $n(\%)$ & $445 / 303(59.5 / 40.5)$ & $97 / 79(55.1 / 44.9)$ & $105 / 69(60.3 / 39.7)$ & $108 / 67(61.7 / 38.3)$ & $135 / 88(60.5 / 39.5)$ & 0.59 \\
\hline Diabetes, $n(\%)$ & $264(35.3)$ & $89(50.6)$ & $64(36.8)$ & $60(34.3)$ & $51(22.9)$ & 0.001 \\
\hline Charlson's score, mean (SD) & $3.7(1.7)$ & $4.3(1.8)$ & $3.7(1.6)$ & $3.6(1.7)$ & $3.3(1.5)$ & 0.001 \\
\hline History of heart disease (any type), $n$ (\%) & $312(41.7)$ & $92(52.3)$ & $69(39.4)$ & $68(38.9)$ & $84(37.7)$ & \\
\hline Coronary heart disease & $194(25.9)$ & $59(33.5)$ & $40(23.0)$ & $39(22.3)$ & $56(25.1)$ & 0.064 \\
\hline Heart failure & 149 (19.9) & $57(32.4)$ & $37(21.3)$ & $31(17.7)$ & $24(10.8)$ & 0.001 \\
\hline Malnutrition, $n(\%)$ & $677 / 49 / 22(90.5 / 6.6 / 2.8)$ & $25(14.2)$ & $16(9.2)$ & $10(5.7)$ & $10(4.4)$ & 0.003 \\
\hline BMI, kg/m², mean (SD) & $25.9(4.8)$ & $25.4(4.4)$ & $25.5(4.6)$ & $26.3(4.4)$ & $26.4(5.3)$ & 0.099 \\
\hline Lean BMI (BIA), $\mathrm{kg} / \mathrm{m}^{2}$, mean (SD) & & & & & & 0.16 \\
\hline Males & $14.0(3.2)$ & $13.5(2.6)$ & $12.8(2.6)$ & $14.1(3.0)$ & $15.1(3.4)$ & \\
\hline Females & $12.2(2.3)$ & $12.5(2.3)$ & $11.4(2.1)$ & $12.4(2.6)$ & $12.4(2.4)$ & \\
\hline Fat mass index (BIA), $\mathrm{kg} / \mathrm{m}^{2}$, mean (SD) & & & & & & 0.90 \\
\hline Males & $13.0(3.6)$ & $12.8(2.4)$ & $13.5(5.1)$ & $12.9(4.2)$ & $12.9(5.2)$ & \\
\hline Females & $13.9(4.6)$ & $14.0(2.8)$ & $12.2(2.4)$ & $15.8(2.8)$ & $13.8(5.1)$ & \\
\hline Overhydration (BIA), L, mean (SD) & $1.28(1.70)$ & $1.68(1.66)$ & $1.44(2.00)$ & $1.64(1.49)$ & $0.85(1.68)$ & 0.06 \\
\hline Ratio ECW/ICW (BIA), mean (SD) & $0.98(0.17)$ & $0.96(0.17)$ & $0.99(0.23)$ & $0.95(0.15)$ & $1.02(0.21)$ & 0.35 \\
\hline GFR, mL/min, mean (SD) & $6.4(3.9)$ & $4.7(3.6)$ & $6.0(3.4)$ & $6.9(3.9)$ & $7.5(4.0)$ & 0.001 \\
\hline Plasma albumin, g/L, mean (SD) & $37.2(5.5)$ & $35.9(5.6)$ & $36.1(5.5)$ & $37.8(5.7)$ & $38.5(5.0)$ & 0.001 \\
\hline Blood hemoglobin, $\mathrm{g} / \mathrm{dL}$, mean (SD) & $10.5(1.7)$ & $10.2(1.7)$ & $10.4(1.6)$ & $10.4(1.5)$ & $10.8(9.7)$ & 0.008 \\
\hline Serum CRP, mg/dL, median (range) & $0.56(0.01-52.4)$ & $0.95(0.06 / 92.4)$ & $0.65(0.02 / 22.30)$ & $0.50(0.04 / 11.36)$ & $0.41(0.01 / 12.10)$ & 0.006 \\
\hline D/P creatinine, $\mathrm{PET}$, mean (SD) & $0.67(0.13)$ & $0.69(0.15)$ & $0.67(0.13)$ & $0.67(0.13)$ & $0.67(0.12)$ & 0.58 \\
\hline UF, baseline PET ( $n=241), \mathrm{mL}, n(\%)$ & $545(286)$ & $454(311)$ & $478(303)$ & $457(285)$ & $537(270)$ & 0.33 \\
\hline Modality of PD (CAPD/APD), $n(\%)$ & $537 / 211(71.8 / 28.2)$ & $126 / 50(71.6 / 28.4)$ & $116 / 58(66.7 / 33.3)$ & $132 / 43(75.4 / 24.6)$ & $163 / 60(73.1 / 26.9)$ & 0.31 \\
\hline Icodextrin, $n(\%)$ & $280(37.4)$ & $74(42.0)$ & $84(48.0)$ & $60(34.3)$ & $62(27.8)$ & 0.001 \\
\hline Loop diuretic dose, mg/day, mean (SD) & $27.8(47.2)$ & $29.4(50.3)$ & $29.2(49.4)$ & $25.9(44.4)$ & $27.1(45.2)$ & 0.88 \\
\hline Loop diuretic therapy, $n(\%)$ & $257(34.4)$ & $60(34.1)$ & $57(32.8)$ & $56(32.0)$ & $77(34.5)$ & 0.89 \\
\hline RAAS antagonists, $n(\%)$ & $214(28.6)$ & $48(27.3)$ & $62(35.6)$ & $47(26.9)$ & $57(25.6)$ & 0.13 \\
\hline Psychotropic drugs, $n(\%)$ & $59(7.8)$ & $22(12.5)$ & $18(10.3)$ & $4(2.3)$ & $13(5.8)$ & 0.018 \\
\hline Immunosuppressive therapy, $n$ (\%) & $59(7.8)$ & $10(5.7)$ & $18(10.3)$ & $14(8.0)$ & $17(7.6)$ & 0.45 \\
\hline
\end{tabular}

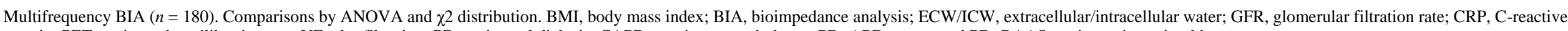
protein; PET, peritoneal equilibration test; UF, ultrafiltration; PD, peritoneal dialysis; CAPD, continuous ambulatory PD; APD, automated PD; RAAS, renin-angiotensin-aldosterone system. 
Before dialysis, $\mathrm{mM} / \mathrm{L}$, mean $(\mathrm{SD}) ; \mathrm{IQR}$

First after initiation of $\mathrm{PD}, \mathrm{mM} / \mathrm{L}$, mean (SD); IQR

Mean of first 3 months, mM/L, mean (SD); IQR

Difference before dialysis - mean of first 3 months, $\mathrm{mM} / \mathrm{L}$, mean (SD)

Decrease $>5 \mathrm{mM} / \mathrm{L}, n(\%)$

ncrease $>5 \mathrm{mM} / \mathrm{L}, n(\%)$

$\mathrm{IQR}$, interquartile range.

Table 3. Correlates of serum sodium levels before initiation of PD (preNa)

\begin{tabular}{|c|c|c|c|c|c|c|}
\hline & \multicolumn{2}{|c|}{ Univariate } & \multirow{2}{*}{$\begin{array}{l}\text { Model } 1 \\
\text { B (95\% CI) } \\
\text { Adjusted } r^{2}=0.118\end{array}$} & \multirow[t]{2}{*}{$p$ value } & \multirow{2}{*}{$\begin{array}{l}\text { Model } 2 \\
\mathrm{~B}(95 \% \mathrm{CI}) \\
\text { Adjusted } r^{2}=0.137\end{array}$} & \multirow[t]{2}{*}{$p$ value } \\
\hline & $r$ & $p$ value & & & & \\
\hline Charlson's score (per point) & -0.09 & 0.016 & $-0.075(-0.131$ to -0.020$)$ & 0.009 & & \\
\hline Diabetes (ref. No.) & -0.09 & 0.028 & & & $-0.21(-0.40$ to -0.03$)$ & 0.019 \\
\hline Heart failure (ref. No.) & -0.18 & $<0.001$ & & & $-0.53(-0.76$ to -0.31$)$ & $<0.001$ \\
\hline Malnutrition (ref. No.) & -0.13 & 0.01 & & & & \\
\hline Plasma albumin (per g/L) & 0.15 & $<0.001$ & $0.014(0.000$ to 0.031$)$ & 0.056 & & \\
\hline GFR (per $\mathrm{mL} / \mathrm{min}$ ) & 0.28 & $<0.001$ & $0.070(0.047$ to 0.093$)$ & $<0.001$ & $0.068(0045$ to 0.091$)$ & $<0.001$ \\
\hline Dose of loop diuretics (per mg/24 h) & 0.13 & 0.001 & $0.002(0.001$ to 0.004$)$ & 0.024 & $0.003(0.001$ to 0.005$)$ & 0.001 \\
\hline Blood hemoglobin (per g/dL) & 0.16 & $<0.001$ & & & & \\
\hline Serum CRP (ref. below median) & -0.13 & 0.003 & & & & \\
\hline
\end{tabular}

Statistics by Spearman's correlation coefficient. ANOVA (univariate) and multiple linear regression analysis (best models with Charlson's score [Model 1] or diabetes and heart

failure [Model 2]). First-order interaction terms not significant in multivariate analysis. Other variables not significant.

$\mathrm{PD}$, peritoneal dialysis; GFR, glomerular filtration rate; CRP, C-reactive protein.

$7.75(4.40) ; 135-140$

$137.74(4.13) ; 135-140$

37.57 (3.60); $135.3-139.8$

$.09(4.35)$

$63(9.3)$ 
Table 4. Correlates of mean serum sodium levels during the first 3 months of PD therapy (mNa)

\begin{tabular}{|c|c|c|c|c|c|c|}
\hline & \multicolumn{2}{|c|}{ Univariate } & \multirow{2}{*}{$\begin{array}{l}\text { Model } 1 \\
\text { B ( } 95 \% \text { CI }) \\
\text { Adjusted } r^{2}=0.191\end{array}$} & \multirow[t]{2}{*}{$p$ value } & \multirow{2}{*}{$\begin{array}{l}\text { Model } 2 \\
\text { B }(95 \% \text { CI }) \\
\text { Adjusted } r^{2}=0.199\end{array}$} & \multirow[t]{2}{*}{$p$ value } \\
\hline & $r$ & $p$ value & & & & \\
\hline Age, years (per year) & 0.08 & 0.041 & & & & \\
\hline Charlson's score (per point) & -0.22 & $<0.0005$ & $-0.121(-0.171$ to -0.069$)$ & $<0.001$ & & \\
\hline Diabetes (ref. No.) & -0.22 & $<0.001$ & & & $-0.37(-0.55$ to -0.20$)$ & $<0.001$ \\
\hline Heart failure (ref. No.) & -0.20 & $<0.001$ & & & $-0.43(-0.63$ to -0.23$)$ & $<0.001$ \\
\hline BMI (per kg/m²) & 0.10 & 0.01 & & & & \\
\hline Malnutrition (ref. No.) & -0.13 & 0.002 & & & & \\
\hline Plasma albumin (per g/L) & 0.21 & $<0.001$ & $0.018(0.003$ to 0.032$)$ & 0.006 & $0.016(0.001$ to 0.030$)$ & 0.018 \\
\hline GFR (per $\mathrm{mL} / \mathrm{min}$ ) & 0.27 & $<0.001$ & $0.082(0.061$ to 0.103$)$ & $<0.001$ & $0.068(0.045$ to 0.091$)$ & $<0.001$ \\
\hline Blood hemoglobin (per g/dL) & 0.12 & 0.001 & $0.069(0.020$ to 0.118$)$ & 0.006 & & \\
\hline Serum CRP (ref. below median) & -0.20 & $<0.001$ & & & & \\
\hline Icodextrin (ref. No.) & -0.15 & 0.001 & $-0.40(-0.56$ to -0.23$)$ & $<0.001$ & $-0.37(-0.54$ to -0.21$)$ & $<0.001$ \\
\hline UF during baseline PET (per $\mathrm{mL}$ ) & 0.12 & 0.007 & & & & \\
\hline
\end{tabular}

Statistics by Spearman's correlation coefficient. ANOVA (univariate) and multiple linear regression analysis (best models with Charlson's score [Model 1] or diabetes and heart failure [Model 2]). First-order interaction terms not significant in multivariate analysis.

Other variables not significant. PD, peritoneal dialysis; $\mathrm{mNa}$, mean serum sodium; BMI, body mass index; GFR, glomerular filtration rate; CRP, C-reactive protein;

UF, ultrafiltration; PET, peritoneal equilibration test. 
Table 5. Correlates of changes in serum sodium levels after initiation of PD

\begin{tabular}{|c|c|c|c|c|}
\hline & \multicolumn{2}{|c|}{ Univariate } & \multirow{2}{*}{$\begin{array}{l}\text { Multivariate } \\
\mathrm{B}(95 \% \mathrm{CI}) \\
\text { Adjusted } r^{2}=0.063\end{array}$} & \multirow[t]{2}{*}{$p$ value } \\
\hline & $r$ & $p$ value & & \\
\hline Age, years (per year) & -0.09 & 0.013 & $-0.004(0.081$ to -0.001$)$ & 0.035 \\
\hline Charlson's score (per point) & -0.11 & 0.013 & & \\
\hline Diabetes (ref. No.) & -0.11 & 0.004 & & \\
\hline Dose of loop diuretics (per mg/24 h) & -0.16 & $<0.001$ & $-0.001(-0.003$ to -0.000$)$ & 0.026 \\
\hline Icodextrin (ref. No.) & -0.19 & $<0.001$ & $-0.25(-0.38$ to -0.12$)$ & $<0.001$ \\
\hline $\mathrm{D} / \mathrm{P}$ creatinine. PET & -0.11 & 0.021 & & \\
\hline UF at $240^{\prime}$ during baseline PET (per mL) & 0.21 & 0.004 & & \\
\hline
\end{tabular}

Statistics by Spearman's correlation coefficient. $\chi 2$ distribution (univariate) and multiple linear regression analysis. First-order interaction terms not significant in multivariate analysis. Other variables not significant. PD, peritoneal dialysis; UF, ultrafiltration; PET, peritoneal equilibration test. 
Univariate Multivariate. Model 1 p value Multivariate. Model 2 p value ( $p$ value $\quad \mathrm{HR}(95 \% \mathrm{CI})$

log rank) -2log likelihood 3,422.2; $\chi^{2} 201.7$

Age, years (per year)

Charlson's score (per point)

Diabetes mellitus (ref. No.)

History of heart failure (ref. No.)

Plasma albumin (per $\mathrm{g} / \mathrm{L}$ )

Blood hemoglobin (per g/dL)

GFR (per $\mathrm{mL} / \mathrm{min}$ )

Malnutrition (ref. No.)

Lean body mass (per $\mathrm{kg} / \mathrm{m}^{2}$ )

Serum CRP (ref. < median)

Immunosuppresive therapy (ref. No.)

RAAS antagonist therapy (ref. No.)

BMI (per $\mathrm{kg} / \mathrm{m}^{2}$ )

$\mathrm{D} / \mathrm{P}$ creatinine at 240 , baseline PET (per point)

UF during baseline PET ( $n=241)$

Ratio ECW/ICW (BIA)

Adjusted effects of study variables

Serum sodium before PD (preNa)(per mM/L)

$\mathrm{mNa}$ (per $\mathrm{mm} / \mathrm{L}$ )

Change in serum sodium levels (per $\mathrm{mm} / \mathrm{L}$ )
$(+)<0.001 \quad 1.05$ (1.04-1.06)
$(+)<0.001 \quad 1.37(1.27-1.47)$
$(+)<0.001$
(+) $<0.001$
$(-)<0.001$
(-) $<0.001$
$0.96(0.94-0.98)$
$0.90(0.83-0.97)$
$(-)<0.001 \quad 0.92(0.89-0.95)$
$(+)<0.001$
(+) 0.004
(+) $<0.001$
(-) 0.044
(-) 0.01
(-) 0.041
(+) $0.013 \quad 1.012(1.001-1.023)$
(-) 0.044
(-) 0.046
$(-)<0.001 \quad 0.97(0.95-1.00)$
$(-)<0.001 \quad 0.96(0.93-0.99)$ $0.67 \quad 1.01(0.76-1.33)$

HR ( $95 \%$ CI)

-2log likelihood 2,605.2;

$\chi^{2} 173.8$

$\begin{array}{rrr}<0.001 & 1.05(1.04-1.07) & <0.001 \\ <0.001 & & \\ & 1.88(1.45-2.45) & <0.001 \\ & 2.07(1.58-2.78) & <0.001 \\ 0.005 & & \\ 0.004 & 0.91(0.84-0.97) & 0.003 \\ <0.001 & 0.94(0.91-0.98) & 0.016\end{array}$

$\begin{array}{ll}1.37(1.06-1.79) & 0.008 \\ 2.04(1.14-3.64) & 0.016\end{array}$

0.034

0.048

$\begin{array}{lll}0.008 & 0.94(0.90-0.98) & 0.007\end{array}$

Statistics by Kaplan-Meier (log rank; univariate) and Cox's regression (in bold, variables included in the best models). For univariate analysis (+) denotes direct and (-) denotes inverse correlation with mortality.

Other variables not significant. First-order interaction terms with $\mathrm{mNa}$ significant for plasma albumin $(p=0.033)$, blood hemoglobin $(p=0.044)$, and GFR $(p=0.029$; Model 1$)$ and for $\operatorname{CRP}(p=0.044)$ and GFR $(p=0.019$; Model 2). Interaction terms for D/P creatinine (Model 1) $p=0.058$ and for blood hemoglobin (Model 2) $p=0.066$.

HR, hazard ratio; GFR, glomerular filtration rate; CRP, C-reactive protein; RAAS, renin-angiotensin-aldosterone system; BMI, body mass index; UF, ultrafiltration; PET, peritoneal equilibration test; ECW/ICW, extracellular/intracellular water; BIA, bioimpedance analysis; PD, peritoneal dialysis; mNa, mean serum sodium. 


\section{Analysis of Factors Modifying the Association between $\mathrm{mNa}$ and Outcome}

Stratified analysis after significant (or close to significance) interaction terms showed that $\mathrm{mNa}$ levels associated a risk of mortality in hypoalbuminemic (but not in normoalbuminemic) patients, as also in patients with higher (but not lower) levels of CRP, higher (but not lower) levels of RKF, lower (but not higher) levels of hemoglobin at inception of PD and faster (but not slower) than median D/Pcrea values (Fig. 2). In addition, the association between $\mathrm{mNa}$ and mortality was apparent only in patients using icodextrin for the long dwell and also those not treated with RAAS antagonists. Despite a patently lower statistical power $(n=180)$, our data also indicated that the association between $\mathrm{mNa}$ and mortality was more evident in patients less overhydrated or presenting lower than median lean body mass levels (Fig. 2). Finally, we observed minor, nonsignificant trends to a better correlation between $\mathrm{mNa}$ and mortality in women ( $p=0.089$ vs. $p$ $=0.17$ in males), malnourished patients ( $p=0.091$ vs. $p=0.19$ in well-nourished patients), and in those treated with continuous ambulatory PD ( $p=0.069$ vs. $p=0.35$ in patients on automated PD; Fig. 3).

To characterize the relatively unexpected patterns of association between $\mathrm{mNa}$ and mortality according to GFR and the use of icodextrin-based solution, we analyzed the correlation between these 2 factors and the main variables scrutinized. As expected, GFR showed a direct correlation with plasma albumin $(p<0.001)$ and hemoglobin $(p<0.001)$ and an inverse correlation with CRP $(p<0.001)$. Diabetics $(p=0.005)$ presented higher levels of GFR, while the opposite was true for patients with malnutrition $(p=0.01)$ or a background of heart failure $(p=0.03)$. On the other hand, patients using icodextrin were more frequently diabetic $(p<0.001)$, had a slightly increased background of heart failure $(p=0.044)$, and presented lower levels of GFR $(p<0.001)$ and plasma albumin $(p<0.001)$, faster peritoneal transport rates $(p<0.001)$, and slightly higher levels of CRP ( $p=0.047)$. Regarding body composition analysis, we observed a limited correlation between a higher extracellular to intracellular water ratio, on one side and a lower GFR $(p=0.052)$ the use of icodextrin $(p=0.045)$ on the other. Finally, lean BMI levels were marginally higher in patients with better preserved GFR $(p=0.078)$.

\section{Discussion}

Hyponatremia is the electrolyte disorder most commonly diagnosed in clinical practice. It can be observed in many different clinical settings and its appearance, particularly if it develops acutely, is a recognized harbinger of poor prognosis [13]. The same contentions apply for patients at different stages of CKD [5, 14, 15], including those treated with hemodialysis [16-19]. The general pathogenesis of this disorder has been extensively investigated and is available for review elsewhere [20]. In the majority of cases, incapacity to regulate adequately water excretion underlies the appearance of this complication, either as the primary culprit or as an important contributor to the generation and persistence of the disorder. Less frequently, other mechanisms may be operative, including abnormal fluid intake rates, presence of osmotically active solutes in extracellular fluid, hypovolemic states, endocrinopathies, or adverse effects of some drugs.

Patients with CKD retain their capacity to excrete excess water until advanced phases of the disorder [11]. As patients approach end-stage CKD, the ability to dilute and concentrate urine vanishes, and isosthenuria becomes the rule. At this point, the capacity of the organism to manage changes in osmolality declines, and the risk of hyponatremia increases [11, 12]. Among hemodialysis patients without significant RKF, hyponatremia is most frequently the consequence of a positive fluid balance (overhydration), but may also be secondary to accumulation of extracellular solutes (e.g., glucose) or transcellular shifts of intracellular solutes, a condition frequently linked to inflammatory and wasting states $[12,17]$. In patients with significant GFR, inflammation may also contribute to hyponatremia by means of IL6-stimulated, nonosmotic secretion of vasopressin [21]. Loss of electrolytes, as observed in malnourished patients [22] or 
those presenting increased intestinal or peritoneal losses, represents a less common cause of hypovolemic hyponatremia, in patients on dialysis.

Individuals undergoing chronic PD share most of the considerations in the preceding paragraph. A better preservation of GFR could help to prevent hypervolemia and, consequently, exert some protective effect against hyponatremia. However, bioimpedance-based surveys have shown than overhydration is very prevalent in these patients, even at PD inception [23]. On the other hand, PD may associate some other characteristics predisposing to hyponatremia, including hyperglycemia and excess peritoneal solute losses. Use of polyglucose-based solutions decreases plasma sodium concentration, seemingly due to water shifts secondary to the accumulation of metabolites of icodextrin [12]. The impact of the latter phenomenon is usually modest, but can be clinically significant in patients with other factors predisposing to hyponatremia [24].

Our study scrutinized serum sodium immediately before PD was started, which provided a reference to evaluate the effect of the introduction of this therapy. Comorbidity and GFR were independent predictors of both preNa and $\mathrm{mNa}$ levels (Tables 3, 4). Prescription of icodextrin was the only factor consistently modifying serum sodium after initiation of PD. Other studies have provided a rather inconsistent list of factors associated with hyponatremia in PD patients, including older age [9, 10], female gender [7, 8], ethnic background [8], diabetes [7, presence of markers of malnutrition and inflammation [12].

The common association of hyponatremia with different systemic conditions feeds the hypothesis that this complication may represent a marker rather than a pathogenic factor of mortality. Moderate chronic hyponatremia (serum $\mathrm{Na}>130 \mathrm{mM} / \mathrm{L}$ ) is usually well tolerated by patients with CKD. However, low serum $\mathrm{Na}$ has been shown to provoke organ and system dysfunctions, including neurologic and cognitive disorders [26], fracture risk, cardiac malfunction, and impaired immunity [12, 20,25]. This question may be consequential because, if we accept a significant direct pathogenic effect, even moderate hyponatremia should become a specific target of management. 
Fig. 1. Survival of patients incident in PD according to the mean of serum sodium levels during the first 3 months of therapy

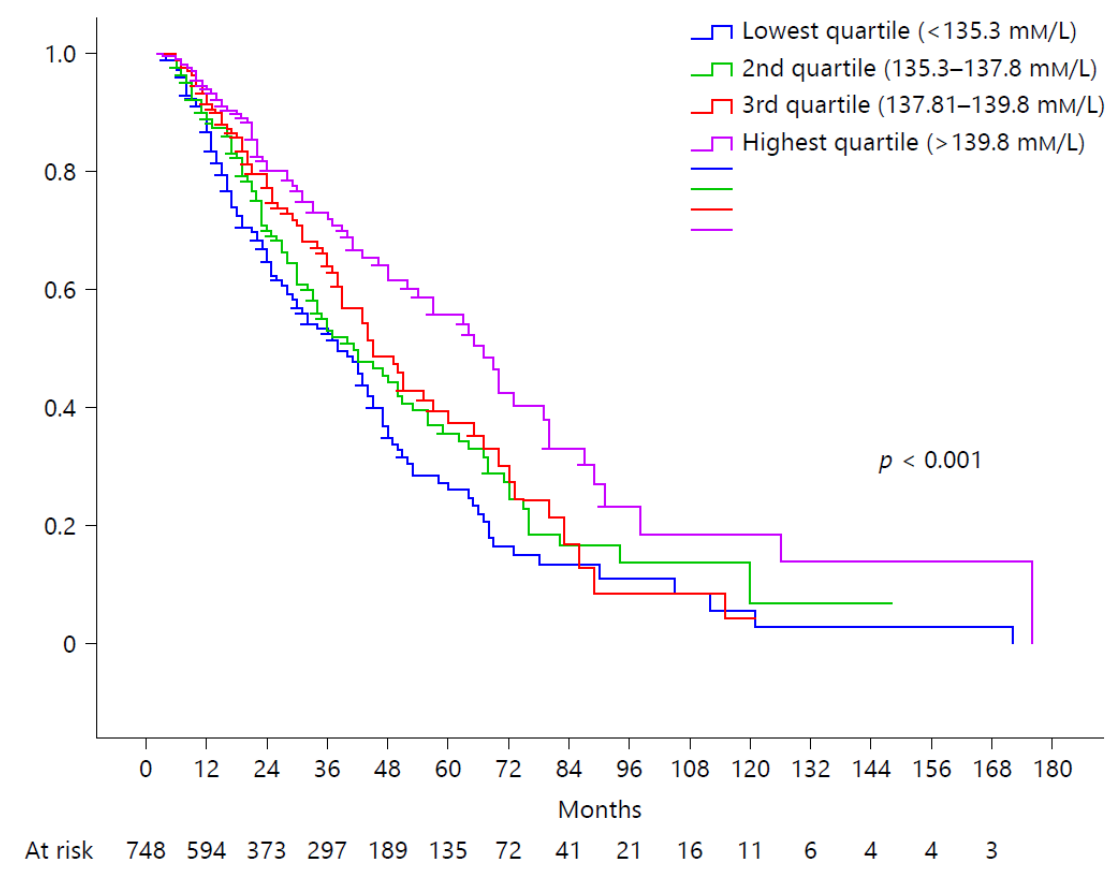

Fig. 2. Foster plot of the association between $\mathrm{mNa}$ levels and patient mortality after stratification of different study variables. Median of lean BMI 13.9 males vs. $12.1(\mathrm{~kg} / \mathrm{m} 2)$ females. ECW, extracellular water; ICW, intracellular water; RAAS, renin-angiotensinaldosterone system; PET, peritoneal equilibration test; GFR, glomerular filtration rate; CRP, Creactive protein; BMI, body mass index.

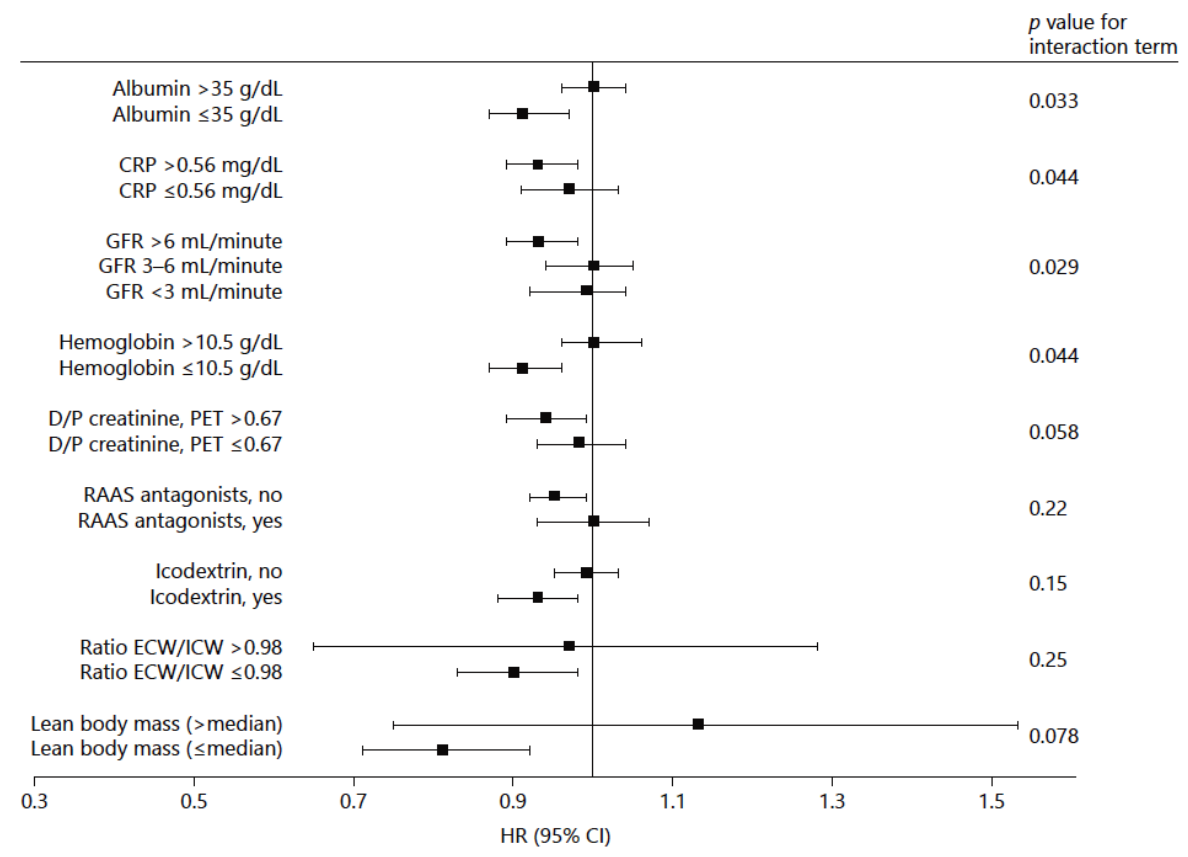


The reported association between hyponatremia and mortality is controversial, in patients undergoing PD. A relatively small size of previous studies may explain why some have been able to disclose such correlation [6,7], which has not been observed by others [9, 10]. In the best powered study to date, Ravel et al. [8] disclosed significant associations between baseline and time-dependent sodium levels, on one side, and later survival of incident PD patients, on the other. Remarkably, the effect varied significantly in the different models applied, emphasizing the difficulties inherent to clarify the prognostic significance of hyponatremia. The study by Ravel et al. [8] scrutinized a wide array of control variables, but could not retrieve some relevant ones, including markers of volume overload, wasting, and inflammation. Moreover, GFR, an essential control variable in this setting, was also estimated from a relatively imprecise marker (renal urea $\mathrm{Kt} / \mathrm{V})[8]$.

Fig. 3. Foster plot of the association between $\mathrm{mNa}$ levels and patient mortality after stratification of demographic and clinical variables (differences not significant). Median of fat mass index 12.4 males vs. 13.1 (kg/m2) females. BMI, body mass index; UF, ultrafiltration; PET, peritoneal equilibration test; PD, peritoneal dialysis; CAPD, continuous ambulatory PD; APD, automated PD.

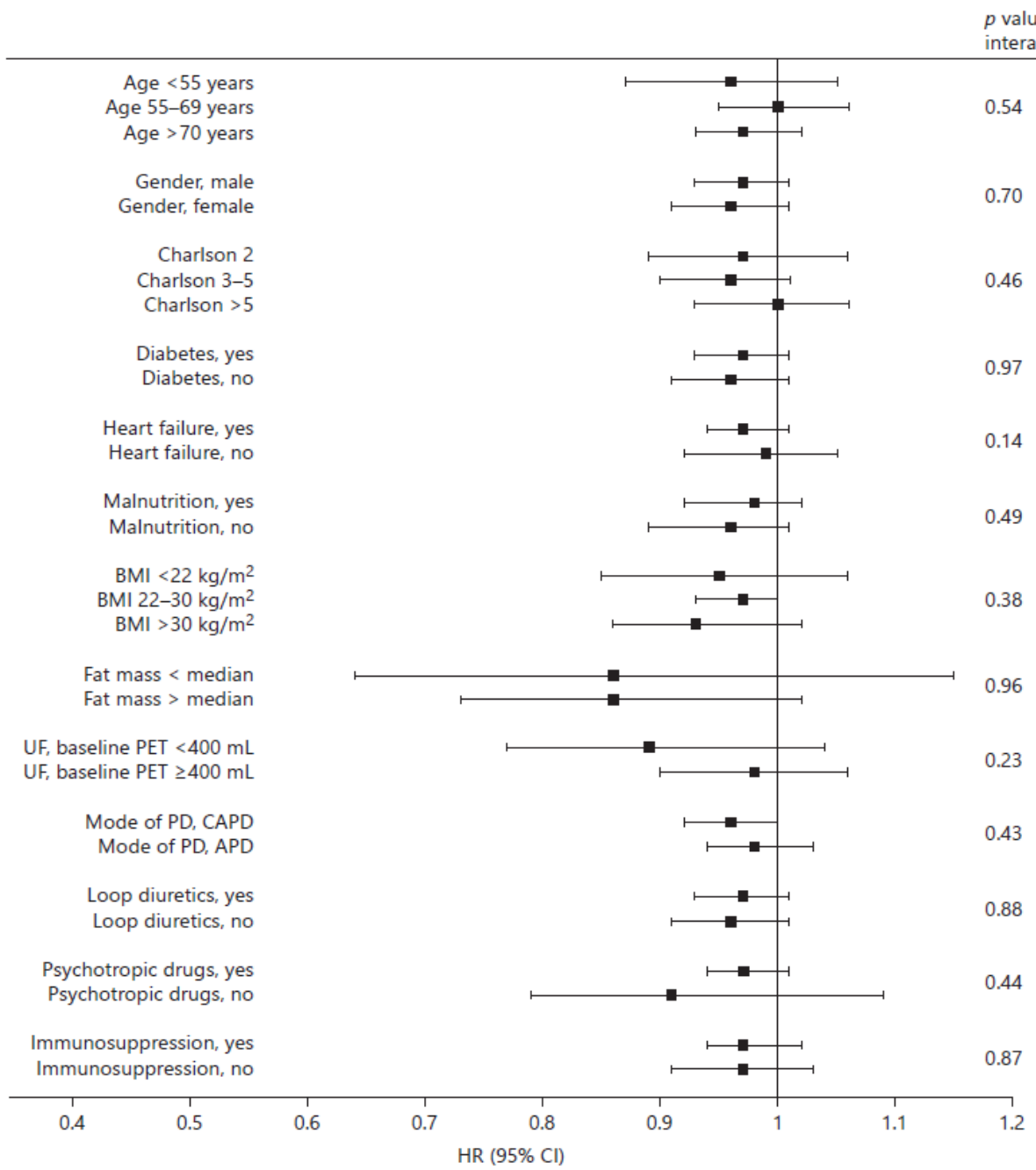


Our results side with previous studies disclosing a significant correlation between serum sodium, on one side, and survival on PD, on the other (Table 6). However, our main objective was to define how this association can differ, according to the putatively dominant factors generating hyponatremia in these patients, namely, overhydration wasting/inflammatory states, and adverse effects of drugs and dialysis prescription. For this purpose, we used stratified analyses (Fig. 2, 3). Our results suggest that hyponatremia predicts mortality mainly in individuals with a background of inflammation and wasting, as shown by direct (elevated CRP protein, lower lean body mass) and indirect (low plasma albumin, low blood hemoglobin, faster peritoneal transport rates, no treatment with RAAS antagonists) markers of this condition. On the contrary, this association was less apparent in settings of mere volume overload (imbalance between fluid intake and removal), which should not be unexpected, because overhydration is a treatable condition. In fact, $\mathrm{mNa}$ was a predictor of mortality in patients with lesser degrees of overhydration by body composition analysis, despite the limited number of cases scrutinized ( $n=180$; Fig. 2$)$. On the other hand, the mode of influence of GFR on the association between $\mathrm{mNa}$ and mortality deserves some comment. On average, patients with higher levels of GFR were less overhydrated, presented less inflammatory features, and were better nourished than their counterparts with lower GFR levels. Our hypothesis is that, in this subset of lower risk of wasting and overhydration, hyponatremia may mark more accurately individuals with particularly poor clinical patterns (including ongoing wasting) demanding an early initiation of renal replacement therapy. The significance of the differing association between $\mathrm{mNa}$ and mortality according to the use of icodextrin to diabetics and patients with lower levels of GFR and a limited capacity of ultrafiltration. These characteristics may match better with the risk of overhydration than with the presence of inflammatory features. However, prescription of this solution aims to prevent overhydration, and is expected to be efficient for this purpose, in a majority of cases. The scenery is further compounded by the particular effect of icodextrin on serum sodium levels (Table 5). Overall, the significance of these findings is unclear, and confounding cannot be excluded.

Generic estimators of malnutrition (including subjective global assessment and BMI) did not exert a clear differential impact on the prognostic significance of hyponatremia, in our study. However, the accuracy of these markers may be confounded by factors such as fat accumulation or overhydration. This limitation may have been aggravated by the relatively low proportion of patients suffering malnutrition (and particularly, severe malnutrition), in our population (Table 1). Remarkably, only 16 of our patients $(2.2 \%)$ presented a BMI $<18 \mathrm{~kg} / \mathrm{m} 2$. A previous, more powered analysis [8] disclosed a limited influence of muscle mass (estimated from serum creatinine) on the pattern of association between serum sodium and patient survival.

Our study suffers some significant limitations, including its retrospective, single-center design. The sample was relatively large, but may still have been underpowered to scrutinize the effects of some variables. Only 180 patients had a body composition analysis, and the capacity of ultrafiltration was estimated in a standardized way in 241 cases. Among the strengths of the study, we should remark the availability of markers of the 3 main conditions resulting in hyponatremia in PD patients, as also the clarity of the results, well supported by the data presented.

In conclusion, our results suggest that hyponatremia performs as a consistent correlate of the risk of mortality in PD patients presenting direct or indirect markers of an underlying wasting and/or inflammatory state. On the contrary, this association is not apparently linked to the presence of mere overhydration, nominal preexisting comorbid conditions, or treatment with loop diuretics or psychotropic drugs. Our findings may provide some support to those contending that, among PD patients, chronic, moderate hyponatremia performs as a marker of the overall risk of mortality rather than as a direct pathogenic factor. Further studies will be necessary to confirm the findings of this single-center analysis. 


\section{Disclosure Statement and Funding Sources}

The authors declare no conflict of interest related to this study. Likewise, we declare that the study did not receive any specific funding.

\section{References}

1. Webster AC, Nagler EV, Morton RL, Masson P. Chronic kidney disease. Lancet. 2017 Mar; 389(10075): 1238-52.

2. Ma L, Zhao S. Risk factors for mortality in patients undergoing hemodialysis: A systematic review and meta-analysis. Int J Cardiol. $2017 \mathrm{Jul} ; 238$ : 151-8.

3. Gilligan S, Raphael KL. Hyperkalemia and Hypokalemia in CKD: Prevalence, Risk Factors, and Clinical Outcomes. Adv Chronic Kidney Dis. 2017 Sep; 24(5): 315-8.

4. Khan S, Floris M, Pani A, Rosner MH. Sodium and volume disorders in advanced chronic kidney disease. Adv Chronic Kidney Dis. 2016 Jul; 23(4): 240-6.

5. Jovanovich A, Berl T. Mortality and serum sodium in CKD-yet another U-shaped curve. Nat Rev Nephrol. 2012 May; 8(5): 261-3.

6. Chang TI, Kim YL, Kim H, Ryu GW, Kang EW, Park JT, et al. Hyponatremia as a predictor of mortality in peritoneal dialysis patients. PLoS One. 2014 Oct; 9(10):e111373.

7. Al-Chidadi A, Nitsch D, Davenport A. The Effect of Serum Sodium on Survival in Patients Treated by Peritoneal Dialysis in the United Kingdom. Perit Dial Int. 2017; 37(1): 70-7.

8. Ravel VA, Streja E, Mehrotra R, Sim JJ, Harley K, Ayus JC, et al. Serum sodium and mortality in a national peritoneal dialysis cohort. Nephrol Dial Transplant. 2017 Jul; 32(7): 1224-33.

9. Kang SH, Cho KH, Park JW, Yoon KW, Do JY. Characteristics and clinical outcomes of hyponatraemia in peritoneal dialysis patients. Nephrology (Carlton). 2013 Feb; 18(2): 1327.

10. Chen KH, Chen CY, Lee CC, Weng CM, Hung CC. Baseline hyponatremia does not predict two-year ortality in patients with chronic peritoneal dialysis. Ren Fail. 2014 Oct; 36(9): 1371-5.

11. Kovesdy CP. Significance of hypo- and hypernatremia in chronic kidney disease. Nephrol Dial Transplant. 2012 Mar; 27(3): 891-8.

12. Rhee CM, Ayus JC, Kalantar-Zadeh K. Hyponatremia in the dialysis population. Kidney Int Rep. 2019 Mar; 4(6): 769-80.

13. Spasovski G, Vanholder R, Allolio B, Annane D, Ball S, Bichet D, et al.; Hyponatraemia Guideline Development Group. Clinical practice guideline on diagnosis and treatment of hyponatraemia. Nephrol Dial Transplant. 2014 Apr; 29(Suppl 2):i1-39.

14. Kovesdy CP, Lott EH, Lu JL, Malakauskas SM, Ma JZ, Molnar MZ, et al. Hyponatremia, hypernatremia, and mortality in patients with chronic kidney disease with and without congestive heart failure. Circulation. $2012 \mathrm{Feb}$; 125(5): 677-84.

15. Chiu DY, Kalra PA, Sinha S, Green D. Association of serum sodium levels with all-cause and cardiovascular mortality in chronic kidney disease: results from a prospective observational study. Nephrology (Carlton). 2016 Jun; 21(6): 476-82.

16. Waikar SS, Curhan GC, Brunelli SM. Mortality associated with low serum sodium concentration in maintenance hemodialysis. Am J Med. 2011 Jan; 124(1): 77-84.

17. Dekker MJ, Marcelli D, Canaud B, Konings CJ, Leunissen KM, Levin NW, et al. Unraveling the relationship between mortality, hyponatremia, inflammation and malnutrition in hemodialysis patients: results from the international MONDO initiative. Eur J Clin Nutr. 2016 Jul; 70(7): 779-84.

18. Rhee CM, Ravel VA, Ayus JC, Sim JJ, Streja E, Mehrotra R, et al. Pre-dialysis serum sodium and mortality in a national incident hemodialysis cohort. Nephrol Dial Transplant. 2016 Jun; 31(6): 992-1001.

19. Perez Garcia R, Palomares I, Merello JI, Ramos R, Maduell F, Molina M, et al. Hiponatremia, mortalidad y hemodialisis: una asociación no explicada. Nefrologia. 2016; 36(1): 42-50. 
20. Verbalis JG. Disorders of water balance. In: Skorecki K, Chertow GM, Marsden PA, Taal MW, Yu AS, editors. Brenner \& Rector's The Kidney Philadelphia: Elsevier; 2016. pp. 460-510.

21. Hoorn EJ, Zietse R. Hyponatremia revisited: translating physiology to practice. Nephron, Physiol. 2008; 108(3): 46-59.

22. Dimitriadis C, Sekercioglu N, Pipili C, Oreopoulos D, Bargman JM. Hyponatremia in peritoneal dialysis: epidemiology in a single center and correlation with clinical and biochemical parameters. Perit Dial Int. 2014 May; 34(3): 260-70.

23. Ronco C, Verger C, Crepaldi C, Pham J, De Los Rios T, Gauly A, et al.; IPOD-PD Study Group. Baseline hydration status in incident peritoneal dialysis patients: the initiative of patient outcomes in dialysis (IPOD-PD study). Nephrol Dial Transplant. 2015 May; 30(5): 849-58.

24. Kunin M, Ganon L, Holtzman EJ, Dinour D. Hyponatremia in refractory congestive heart failure patients treated with icodextrin-based peritoneal dialysis: A case series. Nefrologia.2018 Jan - Feb; 38(1): 87-91.

25. Kim HW, Ryu GW, Park CH, Kang EW, Park JT, Han SH, et al. Hyponatremia Predicts New-Onset Cardiovascular Events in Peritoneal Dialysis Patients. PLoS One. 2015 Jun; 10(6): 0129480.

26. Xu R, Pi HC, Xiong ZY, Liao JL, Hao L, Liu GL, et al. Hyponatremia and cognitive impairment in patients treated with peritoneal dialysis. Clin J Am Soc Nephrol. 2015 Oct; 10(10): 1806-13. 